\title{
TTR
}

Traduction, terminologie, re?daction

\section{Marylin Gaddis Rose. Translation and Literary Criticism. Translation as Analysis. Manchester, St. Jerome Publishing, (Coll. « Translation Theories Explained »), 1997.}

\section{Nicole Côté}

Volume 11, numéro 1, 1er semestre 1998

Diachronie et synchronie

Diachronics and Synchronics

URI : https://id.erudit.org/iderudit/037325ar

DOI : https://doi.org/10.7202/037325ar

Aller au sommaire du numéro

\section{Éditeur(s)}

Association canadienne de traductologie

ISSN

0835-8443 (imprimé)

1708-2188 (numérique)

Découvrir la revue

Citer ce compte rendu

Côté, N. (1998). Compte rendu de [Marylin Gaddis Rose. Translation and Literary Criticism. Translation as Analysis. Manchester, St. Jerome Publishing, (Coll. « Translation Theories Explained »), 1997.] TTR, 11(1), 252-255.

https://doi.org/10.7202/037325ar d'utilisation que vous pouvez consulter en ligne.

https://apropos.erudit.org/fr/usagers/politique-dutilisation/ 


\section{Marylin Gaddis Rose. Translation and Literary Criticism. Translation as Analysis. Manchester, St. Jerome Publishing, (Coll. " Translation Theories Explained "), 1997.}

Si une lecture en diagonale du court ouvrage de Marylin Gaddis Rose il s'agit d'une plaquette, dont la jaquette, au demeurant, est fort belle fatigue en raison de coquilles ${ }^{1}$ choquantes pour les francophones, le lecteur attentif y découvrira toutefois, outre une mine de renseignements sur l'état de la recherche en traductologie, une méthode originale de lecture des œuvres ainsi qu'une analyse sérieuse de traductions d'œuvres canoniques. Toutefois, l'auteure, me semble-t-il, ratisse trop large; les concepts qui sont présentés dans les deux premières sections auraient gagné à être repris et approfondis.

Le but de Gaddis Rose est de démontrer qu'en établissant un pont entre les études traductologiques et la critique littéraire on enrichit sa lecture d'ouvres littéraires. L'auteure affirme que la * lecture stéréoscopique $"^{2}$, qui correspond à l'étude du texte en édition bilingue,

\footnotetext{
I Il y a la très gênante coquille : " Jean-François Lyotard's La Différand » (p. 8; Le Différand), mais aussi des fautes qui, moins évidentes, sont tout de même vite répérées pour peu que l'on ait une bonne connaissance du français : un accent manquant ici et là - * troncs blancs et déperis »un accord oublié $-\alpha$ les murs qui avaient froids" (p. 35; les italiques sont de moi) - ou incorrect $-\propto$ in which case, we should stay with vieux alone or, at most, add gens * (p. 6; il aurait fallu expliquer ici qu'il faut mettre le mot vieux au féminin s'il est suivi de gens : vieilles gens).
}

${ }^{2}$ Traduction littérale de $\times$ stereoscopic reading . 
encourage une lecture de l'œuvre en profondeur et en révèle des aspects insoupçonnés. Postulant que plus l'œuvre est complexe, plus elle est difficile à cemer, Gaddis Rose affirme que l'étude de la traduction de l'œuvre jointe à celle-ci démarque un seuil, un * espace interliminal » (interliminal space) entre l'œuvre originale et la traduction, espace que le traducteur crée en tant qu'auteur et lecteur par procuration.

Gaddis Rose compare l'œuvre à un axe mobile au milieu d'un cercle ou d'un globe dont la circonférence se modifie sans cesse selon les lectures qu'on en fait, cet axe représentant le noyau dur du sens, son * irréductible intégrité ` (p. 5). La circonférence de l'œuvre, qui prend de l'expansion ou se contracte selon les lectures, appartient tout de même au cour de l'œuvre, à son $\propto$ axe $»$, en constituant une partie du potentiel qui s'actualise au fur et à mesure des traductions-lectures. On voit ce que cette vision de la traduction doit à Benjamin. Cette influence n'est pas niée par l'auteure, qui affirme que l'une des époques charnières de la traductologie est le moment où Steiner, dans Après Babel, réhabilite l'essai de Benjamin, * La Tâche du traducteur *. Gaddis Rose favorise ainsi nettement la publication des œuvres avec leur traduction, ce qui oblige, selon elle, le lecteur à réagir à la traduction, en trouvant sa propre traduction, procédé qui redéfinit les limites de l'œuvre. Car entre l'œuvre et sa traduction se trouve le $\alpha$ texte interliminal * qui n'est pas écrit, mais peut être paraphrasé. Cette « interliminalité " serait le don de la traduction aux lecteurs puisqu'elle oblige à une certaine critique, la traduction et la critique littéraires étant toutes deux liées à la production de textes seconds, dérivés. Voilà l'essentiel des deux premières sections de l'ouvrage, intitulées "Postulates of Literary Criticism * et "The Compatibility of Translation and Literary Criticism ».

La troisième, "Ilustrated Historical Overview ", est plus faible en ce qu'elle regroupe par moments des approximations plutôt impressionnistes sur l'évolution de la traduction et sa valeur depuis l'apparition du langage. Toutefois, s'y trouvent aussi des informations précises et intéressantes concernant l'influence des goûts littéraires sur la pratique de la traduction selon les époques. Ainsi, dira l'auteure, « là où il est question de critique littéraire, l'histoire de la traductologie est essentiellement un ensemble de guides successifs visant à satisfaire les 
gô̂ts littéraires d'une époque $\star^{3}$.

La quatrième section, * The Recurring Rhythms ", utilise la métaphore filée du mouvement du pendule qui oscille entre les phases expansives et restrictives d'une culture. Ces phases seraient intimement liées à des approches opposées en traduction : la tendance à accueillir ouvertement la culture étrangère (phase expansive, qui correspondrait à l'approche néolittérale en traduction, c'est-d̀-dire à privilégier le textesource) ou à l'accueillir en en retenant principalement ce qui correspond à sa propre culture (phase restrictive, qui correspondrait à l'approche interprétative en traduction, où l'on privilégie le sens). C'est l'occasion pour l'auteure de dénoncer le conformisme des éditeurs et de certains traducteurs, qui ne font que suivre la norme, d'autant plus que la mise en marché dans le secteur de l'édition ou la censure de l'État influencent le degré de tolérance à l'étranger. En cela, affirme Gaddis Rose, la traduction reflète la phase (restrictive ou expansive) dans laquelle une culture se situe et, plus largement, la variation des normes dans l'histoire de la litterature.

Les trois sections suivantes sont consacrées à des analyses de traduction : Poe traduit par Baudelaire (section 5); Baudelaire traduit par Walter Benjamin et Stefan George (section 6); enfin Proust et Stendhal traduits par Scott Moncrieff (section 7). Ce sont peut-être les sections les mieux réussies du livre, les analyses de traductions constituant visiblement le point fort de Gaddis Rose : les études sont raffinées et complètes, tenant non seulement compte du texte, mais aussi de la traduction officielle (une traduction interlinéale y étant ajoutée) ainsi que du contexte général de production des textes premiers et seconds pour en expliquer les errements, les réussites, en somme, la spécificité.

L'habituelle conclusion est suivie d'une postface où l'auteure justifie tant ses choix de textes (à quand les études de traductions qui ne soient pas canoniques ?) que son approche. Suivent deux autres sections : d'abord, "Appendix on Pedagogy : The Ennui Factor ", qui discute des

${ }^{3}$ Traduction libre de * Where literary criticism is concerned, the history of translation studies is essentially a record of successive guides to pleasing literary taste $\times($ p. 15) 
méthodes utilisées pour éviter l'ennui dans les cours de traduction quoique l'intention soit louable, elle dépasse largement le projet de ce type d'ouvrage -, puis, un glossaire, où tout, des termes * afterlife * et " alexandrine ", en passant par * polysystem * et * postmodernism " jusqu'à " symbolism-decadence ", reçoit une définition qui, pour être courte, $n$ 'en reste pas moins assez complète.

$\mathrm{Si}$ les liens entre les différentes sections sont parfois ténus, si certains concepts sont présentés trop rapidement - en particulier celui d'interliminal space -, l'ouvrage de Gaddis Rose n'en possède pas moins des qualités indéniables : son approche syncrétique de la question, ses très fines études de traductions et des appendices judicieusement commentés plairont à un public varié.

Nicole Côté

CRELIQ, Université Laval 